\title{
Representations of certain binary quadratic forms as Lambert series
}

by

Pee Choon Toh (Singapore)

1. Introduction. Let $q$ be a complex number with $|q|<1$. In [2], A. Berkovich and H. Yesilyurt used Ramanujan's ${ }_{1} \psi_{1}$ summation formula to write two theta series associated with binary quadratic forms as a sum of Lambert series:

$$
\begin{aligned}
\sum_{x, y=-\infty}^{\infty} q^{x^{2}+5 y^{2}} & =1+\sum_{n=1}^{\infty}\left(\frac{-20}{n}\right) \frac{q^{n}}{1-q^{n}}+\sum_{n=1}^{\infty}\left(\frac{n}{5}\right) \frac{q^{n}}{1+q^{2 n}} \\
\sum_{x, y=-\infty}^{\infty} q^{2 x^{2}+2 x y+3 y^{2}} & =1+\sum_{n=1}^{\infty}\left(\frac{-20}{n}\right) \frac{q^{n}}{1-q^{n}}-\sum_{n=1}^{\infty}\left(\frac{n}{5}\right) \frac{q^{n}}{1+q^{2 n}} .
\end{aligned}
$$

By taking the sum and difference of (1.1) and 1.2 , we obtain

$$
\begin{aligned}
& \sum_{x, y=-\infty}^{\infty} q^{x^{2}+5 y^{2}}+\sum_{x, y=-\infty}^{\infty} q^{2 x^{2}+2 x y+3 y^{2}}=2+2 \sum_{n=1}^{\infty}\left(\frac{-20}{n}\right) \frac{q^{n}}{1-q^{n}} \\
& \sum_{x, y=-\infty}^{\infty} q^{x^{2}+5 y^{2}}-\sum_{x, y=-\infty}^{\infty} q^{2 x^{2}+2 x y+3 y^{2}}=2 \sum_{n=1}^{\infty}\left(\frac{n}{5}\right) \frac{q^{n}}{1+q^{2 n}}
\end{aligned}
$$

In general, for an imaginary quadratic field, $K$, of discriminant $d_{K}$ and class number $h$, Dirichlet's theorem [11, Th. 204] states that

$$
h+\omega \sum_{n=1}^{\infty}\left(\frac{d_{K}}{n}\right) \frac{q^{n}}{1-q^{n}}=\sum_{i=1}^{h} \sum_{x, y=-\infty}^{\infty} q^{Q_{i}(x, y)},
$$

where $\omega$ is the number of units and $Q_{i}(x, y)$ are the inequivalent binary quadratic forms of the quadratic field $K$. When $K=\mathbb{Q}(\sqrt{-5})$, we get $(1.3)$. The companion formula (1.4) can be obtained using the theory of genus characters. Thus, identities involving a single Lambert series as a sum of theta series are well understood. What is interesting in this case is that we 
can also isolate each theta series and rewrite it as a sum of Lambert series. This phenomenon is illustrated in the following example involving four theta series. Let $K=\mathbb{Q}(\sqrt{-30})$ and define

$$
F(a, b, c)=\sum_{x, y=-\infty}^{\infty} q^{a x^{2}+b x y+c y^{2}} .
$$

We have

$$
\left[\begin{array}{rrrr}
1 & 1 & 1 & 1 \\
1 & 1 & -1 & -1 \\
1 & -1 & 1 & -1 \\
1 & -1 & -1 & 1
\end{array}\right]\left[\begin{array}{l}
F(1,0,30) \\
F(2,0,15) \\
F(3,0,10) \\
F(5,0,6)
\end{array}\right]=\left[\begin{array}{c}
4+2 \sum_{n=1}^{\infty}\left(\frac{-120}{n}\right) \frac{q^{n}}{1-q^{n}} \\
2 \sum_{n=1}^{\infty}\left(\frac{n}{15}\right) \frac{q^{n}-q^{3 n}}{1+q^{4 n}} \\
2 \sum_{n=1}^{\infty}\left(\frac{10}{n}\right) \frac{q^{n}-q^{2 n}}{1-q^{3 n}} \\
2 \sum_{n=1}^{\infty}\left(\frac{n}{6}\right) \frac{q^{n}-q^{2 n}-q^{3 n}+q^{4 n}}{1-q^{5 n}}
\end{array}\right] .
$$

Observe that the matrix on the left hand side is invertible. Hence, we can rewrite each of the four theta series as a linear combination of four Lambert series.

In the next section, we shall provide a sufficient condition that allows us to express theta series as a linear combination of Lambert series. Explicit examples will be given in Section 3 .

2. Genus characters and a theorem of Kronecker. Let us recall the theory of genus characters [13, pp. 59-62]. Let $K=\mathbb{Q}(\sqrt{N})$ where $N<0$ is a square free integer. The discriminant of $K$ is defined as

$$
d_{K}= \begin{cases}N & \text { if } N \equiv 1(\bmod 4), \\ 4 N & \text { otherwise }\end{cases}
$$

If $d_{K}$ is odd then it can be written as a product of distinct odd primes, $-p_{1} p_{2} \ldots p_{k}$. We set $P_{i}= \pm p_{i}$ so that $d_{K} / P_{i} \equiv 1(\bmod 4)$, and each $d_{K} / P_{i}$ remains an odd discriminant. If $d_{K}$ is even, then either $d_{K}=-8 p_{2} \ldots p_{k}$, where $d_{K} / 4 \equiv 2(\bmod 4)$, or $d_{K}=-4 p_{2} \ldots p_{k}$, where $d_{K} / 4 \equiv 3(\bmod 4)$. In this case, we set $P_{1}$ to be \pm 8 or -4 so that $d_{K} / P_{1}$ remains an odd discriminant.

Let $d_{1}$ be any product of the factors $P_{1}, \ldots, P_{k}$ of $d_{K}$. Then $d_{K}=d_{1} d_{2}$ gives us a decomposition of $d_{K}$ into a product of two coprime discriminants. For each decomposition $d_{K}=d_{1} d_{2}$, and any prime ideal $\mathfrak{p}$ not dividing $d_{K}$, 
we can define a genus character,

$$
\chi(\mathfrak{p})=\chi_{d_{1}}(\mathfrak{p})=\left(\frac{d_{1}}{N(\mathfrak{p})}\right) .
$$

It can be shown [13, p. 60] that $\chi_{d_{1}}=\chi_{d_{2}}$. Hence we can identify $d_{K}=$ $d_{1} d_{2}=d_{2} d_{1}$, giving us a total of $2^{k-1}$ decompositions.

If $\mathfrak{p} \mid d_{K}$, then one of $\chi_{d_{1}}, \chi_{d_{2}}$ is zero and the other is non-zero; we then take $\chi$ to be the non-zero value. There are $2^{k-1}$ different genus characters corresponding to decompositions of $d_{K}$. In fact, the genus characters form an abelian group, $\mathfrak{G}$, of order $2^{k-1}[13$, p. 66].

Now, for an imaginary quadratic field $K$ [9, p. 190], we have

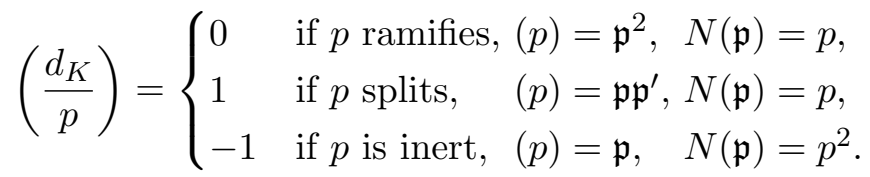

If $\chi$ is a genus character (corresponding to $d_{K}=d_{1} d_{2}$ ), then the $L$-series equals

$$
\begin{aligned}
& L_{K}(s, \chi)=\sum_{\text {ideal } \mathfrak{a}} \frac{\chi(\mathfrak{a})}{N(\mathfrak{a})^{s}}=\prod_{\mathfrak{p} \text { prime }}\left(1-\frac{\chi(\mathfrak{p})}{N(\mathfrak{p})^{s}}\right)^{-1} \\
= & \prod_{\substack{\mathfrak{p} \text { over } \\
\text { ramified } p}}\left(1-\frac{\chi(\mathfrak{p})}{N(\mathfrak{p})^{s}}\right)^{-1} \prod_{\substack{\text { pover } \\
\text { split } p}}\left(1-\frac{\chi(\mathfrak{p})}{N(\mathfrak{p})^{s}}\right)^{-1} \prod_{\substack{\mathfrak{p} \text { over } \\
\text { inert } p}}\left(1-\frac{\chi(\mathfrak{p})}{N(\mathfrak{p})^{s}}\right)^{-1} .
\end{aligned}
$$

For the first product, we may assume without loss of generality that $p \mid d_{2}$, hence $\chi(\mathfrak{p})=\left(\frac{d_{1}}{p}\right)$ and $\left(\frac{d_{2}}{p}\right)=0$. Thus,

$$
\begin{aligned}
\prod_{\substack{\mathfrak{p} \text { over } \\
\text { ramified } p}} & \left(1-\frac{\chi(\mathfrak{p})}{N(\mathfrak{p})^{s}}\right)^{-1} \\
& =\prod_{\operatorname{ramified} p}\left(1-\left(\frac{d_{1}}{p}\right) p^{-s}\right)^{-1} \prod_{\operatorname{ramified} p}\left(1-\left(\frac{d_{2}}{p}\right) p^{-s}\right)^{-1} .
\end{aligned}
$$

In the second product, since $\chi_{d_{1}}=\chi_{d_{2}}$ and $p$ lies under $\mathfrak{p}$ and $\mathfrak{p}^{\prime}$,

$$
\begin{aligned}
\prod_{\substack{\mathfrak{p} \text { over } \\
\text { split } p}}\left(1-\frac{\chi(\mathfrak{p})}{N(\mathfrak{p})^{s}}\right)^{-1} & \\
& =\prod_{\text {split } p}\left(1-\left(\frac{d_{1}}{p}\right) p^{-s}\right)^{-1} \prod_{\text {split } p}\left(1-\left(\frac{d_{1}}{p}\right) p^{-s}\right)^{-1} \\
& =\prod_{\text {split } p}\left(1-\left(\frac{d_{1}}{p}\right) p^{-s}\right)^{-1} \prod_{\text {split } p}\left(1-\left(\frac{d_{2}}{p}\right) p^{-s}\right)^{-1} .
\end{aligned}
$$


For the last product, $\chi(\mathfrak{p})=\left(\frac{d_{1}}{p^{2}}\right)=1$. However, since $\left(\frac{d_{K}}{p}\right)=\left(\frac{d_{1}}{p}\right)\left(\frac{d_{2}}{p}\right)$ $=-1$, we have

$$
\begin{aligned}
& \prod_{\substack{\mathfrak{p} \text { over } \\
\text { inert } p}}\left(1-\frac{\chi(\mathfrak{p})}{N(\mathfrak{p})^{s}}\right)^{-1}=\prod_{\text {inert } p}\left(1-p^{-2 s}\right)^{-1} \\
& \quad=\prod_{\text {inert } p}\left(1-p^{-s}\right)^{-1}\left(1+p^{-s}\right)^{-1} \\
& =\prod_{\text {inert } p}\left(1-\left(\frac{d_{1}}{p}\right) p^{-s}\right)^{-1} \prod_{\text {inert } p}\left(1-\left(\frac{d_{2}}{p}\right) p^{-s}\right)^{-1}
\end{aligned}
$$

Combining these, we obtain Kronecker's theorem [13, p. 62],

$$
\begin{aligned}
L_{K}(s, \chi) & =\prod_{p}\left(1-\left(\frac{d_{1}}{p}\right) p^{-s}\right)^{-1} \prod_{p}\left(1-\left(\frac{d_{2}}{p}\right) p^{-s}\right)^{-1} \\
& =L_{d_{1}}(s) L_{d_{2}}(s) .
\end{aligned}
$$

Next, applying the inverse Mellin transform to 2.5), we get

$$
\begin{aligned}
\sum_{\mathfrak{a} \neq 0} \chi(\mathfrak{a}) q^{N(\mathfrak{a})} & =\left(\sum_{n=1}^{\infty}\left(\frac{d_{1}}{n}\right) q^{n}\right)\left(\sum_{n=1}^{\infty}\left(\frac{d_{2}}{n}\right) q^{n}\right) \\
& =\sum_{n=1}^{\infty}\left(\frac{d_{1}}{n}\right) \frac{\sum_{k=1}^{\left|d_{2}\right|}\left(\frac{d_{2}}{k}\right) q^{k n}}{1-q^{\left|d_{2}\right| n}} .
\end{aligned}
$$

Using $\mathcal{L}_{d_{1}, d_{2}}(q)$ to denote the last sum, we can now state our result.

THEOREM 2.1. Let $K$ be an imaginary quadratic field, with $\omega$ units, where the ideal class group is isomorphic to the group $\mathfrak{G}$ of genus characters. Let $a x^{2}+b x y+c y^{2}$ be a primitive binary quadratic form with discriminant $d_{K}$. If $p$ is any unramified prime represented by this form, then

$$
\begin{aligned}
F(a, b, c) & =1+\frac{\omega}{|\mathfrak{G}|} \sum_{\chi_{d_{1}} \in \mathfrak{G}} \chi_{d_{1}}(p) \mathcal{L}_{d_{1}, d_{2}}(q) \\
& =1+\frac{\omega}{|\mathfrak{G}|} \sum_{\text {admissible } d_{1}}\left(\frac{d_{1}}{p}\right) \mathcal{L}_{d_{1}, d_{2}}(q) .
\end{aligned}
$$

Proof. Let $m=|\mathfrak{G}|$. Under the hypothesis of the theorem, there is an isomorphism from the form class group of primitive binary quadratic forms to the ideal class group [6, p. 113], which is hence isomorphic to $\mathfrak{G}$. Next, let $Q_{i}(x, y)$ be the representatives of the form class group and $F_{i}=\sum q^{Q_{i}(x, y)}$ be the associated theta series, where the sum is over all integers $x$ and $y$, excluding $(x, y)=(0,0)$. Then 2.6) gives us a system of $m$ equations of the 
form

$$
\omega \mathcal{L}_{d_{1}, d_{2}}(q)=\left(\frac{d_{1}}{n_{1}}\right) F_{1}+\left(\frac{d_{1}}{n_{2}}\right) F_{2}+\cdots+\left(\frac{d_{1}}{n_{m}}\right) F_{m},
$$

where $n_{i}$ is any integer represented by $Q_{i}(x, y)$, coprime to $d_{K}$. Hence we have the matrix equation

$$
\omega \hat{L}=A \hat{F} .
$$

Here $\hat{F}=\left(F_{1}, \ldots, F_{k}\right), \hat{L}$ is the vector of the Lambert series $\mathcal{L}_{d_{1}, d_{2}}(q)$, and $A$ is the character table for $\mathfrak{G}$. By the orthogonality relations, [1, Ch. 6], we can recover each $F_{j}$ as

$$
F_{j}=\frac{\omega}{m} \sum_{\chi_{d_{1}} \in \mathfrak{G}} \chi_{d_{1}}\left(n_{j}\right) \mathcal{L}_{d_{1}, d_{2}}(q) .
$$

The proof is complete when we add the case $(x, y)=(0,0)$ and replace $n_{j}$ by a suitable prime.

The condition in Theorem 2.1 is commonly described as imaginary quadratic fields having one class per genus. S. Chowla [4] proved that there are finitely many such fields. Further work by J. D. Swift [15], S. Chowla and W. E. Briggs [5], E. Grosswald [7] and P. J. Weinberger [16], showed that besides the 65 that are currently known, there exists at most one more field with one class per genus.

Theorem 2.1 can easily be generalized to imaginary quadratic fields with more than one class per genus. However, in this case, it is not possible to isolate the several theta series associated to the same genus and the corresponding result is not as striking.

There are another 36 known form class groups that have one class per genus. These are said to have nonfundamental discriminants and correspond to orders [6, p. 132] rather than the ring of integers in the imaginary quadratic field. Theorem 2.1 does not apply to these 36 .

For these 101 discriminants, N. A. Hall [8] has computed explicit formulas for the number of representations of an integer by binary quadratic forms, while K. S. Williams [17] has given identities analogous to (1.5).

For recent work on the problem of representations of an integer by binary quadratic forms (not necessarily having one class per genus), see Z. H. Sun and K. S. Williams [14, P. Kaplan and K. S. Williams [10] and references therein.

3. Examples and tables. There are exactly nine imaginary quadratic fields with class number $h=1$, listed according to their discriminants:

$$
d_{K}=-3,-4,-7,-8,-11,-19,-43,-67,-163 .
$$

L. C. Shen [12] has given explicit identities for these cases. 
There are 18 imaginary quadratic fields with class number $h=2$, listed according to their discriminants:

$$
\begin{aligned}
d_{K}= & -15,-20,-24,-35,-40,-51,-52,-88,-91 \\
& -115,-123,-148,-187,-232,-235,-267,-403,-427 .
\end{aligned}
$$

In the cases where $d_{K}=-4 \ell, \ell=5,13$ or 37 , we have

$$
\sum_{x, y=-\infty}^{\infty} q^{x^{2}+\ell y^{2}}=1+\sum_{n=1}^{\infty}\left(\frac{-4 \ell}{n}\right) \frac{q^{n}}{1-q^{n}}+\sum_{n=1}^{\infty}\left(\frac{\ell}{n}\right) \frac{q^{n}}{1+q^{2 n}},
$$

$$
\sum_{x, y=-\infty}^{\infty} q^{2 x^{2}+2 x y+\frac{\ell+1}{2} y^{2}}=1+\sum_{n=1}^{\infty}\left(\frac{-4 \ell}{n}\right) \frac{q^{n}}{1-q^{n}}-\sum_{n=1}^{\infty}\left(\frac{\ell}{n}\right) \frac{q^{n}}{1+q^{2 n}} .
$$

(3.3) and (3.4) were established by H. H. Chan and S. H. Chan [3].

When $d_{K}=-8 \ell, \ell=3,5,11$ or 29 ,

$$
\begin{aligned}
\sum_{x, y=-\infty}^{\infty} q^{x^{2}+2 \ell y^{2}} & =1+\sum_{n=1}^{\infty}\left(\frac{-8 \ell}{n}\right) \frac{q^{n}}{1-q^{n}}+\sum_{n=1}^{\infty}\left(\frac{n}{\ell}\right) \frac{q^{n}+\left(\frac{-1}{\ell}\right) q^{3 n}}{1+q^{4 n}}, \\
\sum_{x, y=-\infty}^{\infty} q^{2 x^{2}+\ell y^{2}} & =1+\sum_{n=1}^{\infty}\left(\frac{-8 \ell}{n}\right) \frac{q^{n}}{1-q^{n}}-\sum_{n=1}^{\infty}\left(\frac{n}{\ell}\right) \frac{q^{n}+\left(\frac{-1}{\ell}\right) q^{3 n}}{1+q^{4 n}} .
\end{aligned}
$$

The case of $\ell=3$ was also given in [2].

The remaining discriminants are all of the form $d_{K}=-\ell m$, according to the following table:

$$
\begin{aligned}
& \begin{array}{cccccc}
\hline \ell & m & D=\ell m & a & b & c \\
\hline 3 & 5 & 15 & 2 & 1 & 2
\end{array} \\
& \begin{array}{llllll}
7 & 5 & 35 & 3 & 1 & 3
\end{array} \\
& \begin{array}{llllll}
3 & 17 & 51 & 3 & 3 & 5
\end{array} \\
& \begin{array}{llllll}
7 & 13 & 91 & 5 & 3 & 5
\end{array} \\
& \begin{array}{llllll}
23 & 5 & 115 & 5 & 5 & 7
\end{array} \\
& \begin{array}{llllll}
3 & 41 & 123 & 3 & 3 & 11
\end{array} \\
& \begin{array}{llllll}
11 & 17 & 187 & 7 & 3 & 7
\end{array} \\
& \begin{array}{llllll}
47 & 5 & 235 & 5 & 5 & 13
\end{array} \\
& \begin{array}{llllll}
3 & 89 & 267 & 3 & 3 & 23
\end{array} \\
& \begin{array}{llllll}
31 & 13 & 403 & 11 & 9 & 11
\end{array} \\
& \begin{array}{llllll}
61 & 7 & 427 & 7 & 7 & 17 \\
\hline
\end{array} \\
& \sum_{x, y=-\infty}^{\infty} q^{x^{2}+x y+\frac{D+1}{4} y^{2}}=1+\sum_{n=1}^{\infty}\left(\frac{-D}{n}\right) \frac{q^{n}}{1-q^{n}}+\sum_{n=1}^{\infty}\left(\frac{-\ell}{n}\right) \frac{\sum_{j=1}^{m-1}\left(\frac{j}{m}\right) q^{j n}}{1-q^{m n}}, \\
& \sum_{x, y=-\infty}^{\infty} q^{a x^{2}+b x y+c y^{2}}=1+\sum_{n=1}^{\infty}\left(\frac{-D}{n}\right) \frac{q^{n}}{1-q^{n}}-\sum_{n=1}^{\infty}\left(\frac{-\ell}{n}\right) \frac{\sum_{j=1}^{m-1}\left(\frac{j}{m}\right) q^{j n}}{1-q^{m n}} .
\end{aligned}
$$


There are 24 imaginary quadratic fields with class number $h=4$, having one class per genus. We give a table listing the discriminants together with the representatives of the form class group, as well as one example for $d_{K}=$ -84 .

\begin{tabular}{lllll}
\hline$d_{k}$ & \multicolumn{4}{c}{$F(a, b, c)$} \\
\hline-84 & $(1,0,21)$ & $(2,2,11)$ & $(3,0,7)$ & $(5,4,5)$ \\
-120 & $(1,0,30)$ & $(2,0,15)$ & $(3,0,10)$ & $(5,0,6)$ \\
-132 & $(1,0,33)$ & $(2,2,17)$ & $(3,0,11)$ & $(6,6,7)$ \\
-168 & $(1,0,42)$ & $(2,0,21)$ & $(3,0,14)$ & $(6,0,7)$ \\
-195 & $(1,1,49)$ & $(3,3,17)$ & $(5,5,11)$ & $(7,1,7)$ \\
-228 & $(1,0,57)$ & $(2,2,29)$ & $(3,0,19)$ & $(6,6,11)$ \\
-280 & $(1,0,70)$ & $(2,0,35)$ & $(5,0,14)$ & $(7,0,10)$ \\
-312 & $(1,0,78)$ & $(2,0,39)$ & $(3,0,26)$ & $(6,0,13)$ \\
-340 & $(1,0,85)$ & $(2,2,43)$ & $(5,0,17)$ & $(10,10,11)$ \\
-372 & $(1,0,93)$ & $(2,2,47)$ & $(3,0,31)$ & $(6,6,17)$ \\
-408 & $(1,0,102)$ & $(2,0,51)$ & $(3,0,34)$ & $(6,0,17)$ \\
-435 & $(1,1,109)$ & $(3,3,37)$ & $(5,5,23)$ & $(11,7,11)$ \\
-483 & $(1,1,121)$ & $(3,3,41)$ & $(7,7,19)$ & $(11,1,11)$ \\
-520 & $(1,0,130)$ & $(2,0,65)$ & $(5,0,26)$ & $(10,0,13)$ \\
-532 & $(1,0,133)$ & $(2,2,67)$ & $(7,0,19)$ & $(13,12,13)$ \\
-555 & $(1,1,139)$ & $(3,3,47)$ & $(5,5,29)$ & $(13,11,13)$ \\
-595 & $(1,1,149)$ & $(5,5,31)$ & $(7,7,23)$ & $(13,9,13)$ \\
-627 & $(1,1,157)$ & $(3,3,53)$ & $(11,11,17)$ & $(13,7,13)$ \\
-708 & $(1,0,177)$ & $(2,2,89)$ & $(3,0,59)$ & $(6,6,31)$ \\
-715 & $(1,1,179)$ & $(5,5,37)$ & $(11,11,19)$ & $(13,13,17)$ \\
-760 & $(1,0,190)$ & $(2,0,95)$ & $(5,0,38)$ & $(10,0,19)$ \\
-795 & $(1,1,199)$ & $(3,3,67)$ & $(5,5,41)$ & $(15,15,17)$ \\
-1012 & $(1,0,253)$ & $(2,2,127)$ & $(11,0,23)$ & $(17,12,17)$ \\
-1435 & $(1,1,359)$ & $(5,5,73)$ & $(7,7,53)$ & $(19,3,19)$ \\
\hline
\end{tabular}

For $d_{K}=-84$, we set

$$
\begin{array}{ll}
L_{1}=\sum_{n=1}^{\infty}\left(\frac{-84}{n}\right) \frac{q^{n}}{1-q^{n}}, \quad L_{2}=\sum_{n=1}^{\infty}\left(\frac{28}{n}\right) \frac{q^{n}-q^{2 n}}{1-q^{3 n}}, \\
L_{3}=\sum_{n=1}^{\infty}\left(\frac{n}{7}\right) \frac{q^{n}-q^{5 n}}{1+q^{6 n}}, \quad L_{4}=\sum_{n=1}^{\infty}\left(\frac{21}{n}\right) \frac{q^{n}}{1+q^{2 n}} .
\end{array}
$$

Then

$$
\sum_{x, y=-\infty}^{\infty} q^{x^{2}+21 y^{2}}=1+\frac{1}{2}\left(L_{1}+L_{2}+L_{3}+L_{4}\right),
$$




$$
\begin{aligned}
\sum_{x, y=-\infty}^{\infty} q^{3 x^{2}+7 y^{2}} & =1+\frac{1}{2}\left(L_{1}+L_{2}-L_{3}-L_{4}\right), \\
\sum_{x, y=-\infty}^{\infty} q^{2 x^{2}+2 x y+11 y^{2}} & =1+\frac{1}{2}\left(L_{1}-L_{2}+L_{3}-L_{4}\right), \\
\sum_{x, y=-\infty}^{\infty} q^{5 x^{2}+4 x y+5 y^{2}} & =1+\frac{1}{2}\left(L_{1}-L_{2}-L_{3}+L_{4}\right) .
\end{aligned}
$$

\begin{tabular}{|c|c|c|c|c|}
\hline \multirow{2}{*}{$\begin{array}{c}d_{k} \\
-420\end{array}$} & \multicolumn{4}{|c|}{$F(a, b, c)$} \\
\hline & $(1,0,105)$ & $(2,2,53)$ & $(3,0,35)$ & $(5,0,21)$ \\
\hline & $(6,6,19)$ & $(7,0,15)$ & $(10,10,13)$ & $(11,8,11)$ \\
\hline \multirow[t]{2}{*}{-660} & $(1,0,165)$ & $(2,2,83)$ & $(3,0,55)$ & $(5,0,33)$ \\
\hline & $(6,6,29)$ & $(10,10,19)$ & $(11,0,15)$ & $(13,4,13)$ \\
\hline \multirow[t]{2}{*}{-840} & $(1,0,210)$ & $(2,0,105)$ & $(3,0,70)$ & $(5,0,42)$ \\
\hline & $(6,0,35)$ & $(7,0,30)$ & $(10,0,21)$ & $(14,0,15)$ \\
\hline \multirow[t]{2}{*}{-1092} & $(1,0,273)$ & $(2,2,137)$ & $(3,0,91)$ & $(6,6,47)$ \\
\hline & $(7,0,39)$ & $(13,0,21)$ & $(14,14,23)$ & $(17,8,17)$ \\
\hline \multirow[t]{2}{*}{-1155} & $(1,1,289)$ & $(3,3,97)$ & $(5,5,59)$ & $(7,7,43)$ \\
\hline & $(11,11,29)$ & $(15,15,23)$ & $(17,1,17)$ & $(19,17,19)$ \\
\hline \multirow[t]{2}{*}{-1320} & $(1,0,330)$ & $(2,0,165)$ & $(3,0,110)$ & $(5,0,66)$ \\
\hline & $(6,0,55)$ & $(10,0,33)$ & $(11,0,30)$ & $(15,0,22)$ \\
\hline \multirow[t]{2}{*}{-1380} & $(1,0,345)$ & $(2,2,173)$ & $(3,0,115)$ & $(5,0,69)$ \\
\hline & $(6,6,59)$ & $(10,10,37)$ & $(15,0,23)$ & $(19,8,19)$ \\
\hline \multirow[t]{2}{*}{-1428} & $(1,0,357)$ & $(2,2,179)$ & $(3,0,119)$ & $(6,6,61)$ \\
\hline & $(7,0,51)$ & $(14,14,29)$ & $(17,0,21)$ & $(19,4,19)$ \\
\hline \multirow[t]{2}{*}{-1540} & $(1,0,385)$ & $(2,2,193)$ & $(5,0,77)$ & $(7,0,55)$ \\
\hline & $(10,10,41)$ & $(11,0,35)$ & $(14,14,31)$ & $(22,22,23)$ \\
\hline \multirow[t]{2}{*}{-1848} & $(1,0,462)$ & $(2,0,231)$ & $(3,0,154)$ & $(6,0,77)$ \\
\hline & $(7,0,66)$ & $(11,0,42)$ & $(14,0,33)$ & $(21,0,22)$ \\
\hline \multirow[t]{2}{*}{-1995} & $(1,1,499)$ & $(3,3,167)$ & $(5,5,101)$ & $(7,7,73)$ \\
\hline & $(15,15,37)$ & $(19,19,31)$ & $(21,21,29)$ & $(23,11,23)$ \\
\hline \multirow[t]{2}{*}{-3003} & $(1,1,751)$ & $(3,3,251)$ & $(7,7,109)$ & $(11,11,71)$ \\
\hline & $(13,13,61)$ & $(21,21,41)$ & $(29,19,29)$ & $(31,29,31)$ \\
\hline \multirow[t]{2}{*}{-3315} & $(1,1,829)$ & $(3,3,277)$ & $(5,5,167)$ & $(13,13,67)$ \\
\hline & $(15,15,59)$ & $(17,17,53)$ & $(29,7,29)$ & $(31,23,31)$ \\
\hline
\end{tabular}

For imaginary quadratic fields with class number $h=8$, the following 13 have one class per genus:

We illustrate Theorem 2.1 by writing $F(1,0,105)$ in terms of eight Lambert series: 


$$
\begin{aligned}
\sum_{x, y=-\infty}^{\infty} q^{x^{2}+105 y^{2}} & =1+\frac{1}{4}\left(\sum_{n=1}^{\infty}\left(\frac{-420}{n}\right) \frac{q^{n}}{1-q^{n}}+\sum_{n=1}^{\infty}\left(\frac{105}{n}\right) \frac{q^{n}}{1+q^{2 n}}\right. \\
& +\sum_{n=1}^{\infty}\left(\frac{140}{n}\right) \frac{q^{n}-q^{2 n}}{1-q^{3 n}}+\sum_{n=1}^{\infty}\left(\frac{-84}{n}\right) \frac{\sum_{k=1}^{5}\left(\frac{5}{k}\right) q^{k n}}{1-q^{5 n}} \\
& +\sum_{n=1}^{\infty}\left(\frac{60}{n}\right) \frac{\sum_{k=1}^{7}\left(\frac{-7}{k}\right) q^{k n}}{1-q^{7 n}}+\sum_{n=1}^{\infty}\left(\frac{-35}{n}\right) \frac{\sum_{k=1}^{12}\left(\frac{12}{k}\right) q^{k n}}{1-q^{12 n}} \\
& \left.+\sum_{n=1}^{\infty}\left(\frac{28}{n}\right) \frac{\sum_{k=1}^{15}\left(\frac{-15}{k}\right) q^{k n}}{1-q^{15 n}}+\sum_{n=1}^{\infty}\left(\frac{-20}{n}\right) \frac{\sum_{k=1}^{21}\left(\frac{21}{k}\right) q^{k n}}{1-q^{21 n}}\right) .
\end{aligned}
$$

Finally for imaginary quadratic fields with class number $h=16,-5460$ is the only fundamental discriminant with one class per genus.

\begin{tabular}{lllll}
\hline \multicolumn{1}{c}{$d_{k}$} & \multicolumn{4}{c}{$F(a, b, c)$} \\
\hline-5460 & $(1,0,1365)$ & $(2,2,683)$ & $(3,0,455)$ & $(5,0,273)$ \\
& $(6,6,229)$ & $(7,0,195)$ & $(10,10,139)$ & $(13,0,105)$ \\
& $(14,14,101)$ & $(15,0,91)$ & $(21,0,65)$ & $(26,26,59)$ \\
& $(30,30,53)$ & $(35,0,39)$ & $(37,4,37)$ & $(42,42,43)$ \\
\hline
\end{tabular}

We again illustrate Theorem 2.1 by writing $F(37,4,37)$ in terms of sixteen Lambert series:

$$
\begin{array}{rl}
\sum_{x, y=-\infty}^{\infty} q^{37 x^{2}+4 x y+37 y^{2}} & 1+\frac{1}{8}\left(\sum_{n=1}^{\infty}\left(\frac{-5460}{n}\right) \frac{q^{n}}{1-q^{n}}+\sum_{n=1}^{\infty}\left(\frac{1365}{n}\right) \frac{q^{n}}{1+q^{2 n}}\right. \\
= & \sum_{n=1}^{\infty}\left(\frac{1820}{n}\right) \frac{q^{n}-q^{2 n}}{1-q^{3 n}}-\sum_{n=1}^{\infty}\left(\frac{-1092}{n}\right) \frac{\sum_{k=1}^{5}\left(\frac{5}{k}\right) q^{k n}}{1-q^{5 n}} \\
& +\sum_{n=1}^{\infty}\left(\frac{780}{n}\right) \frac{\sum_{k=1}^{7}\left(\frac{-7}{k}\right) q^{k n}}{1-q^{7 n}}-\sum_{n=1}^{\infty}\left(\frac{-420}{n}\right) \frac{\sum_{k=1}^{13}\left(\frac{13}{k}\right) q^{k n}}{1-q^{13 n}} \\
& +\sum_{n=1}^{\infty}\left(\frac{-455}{n}\right) \frac{\sum_{k=1}^{12}\left(\frac{12}{k}\right) q^{k n}}{1-q^{12 n}}-\sum_{n=1}^{\infty}\left(\frac{364}{n}\right) \frac{\sum_{k=1}^{15}\left(\frac{-15}{k}\right) q^{k n}}{1-q^{15 n}} \\
& -\sum_{n=1}^{\infty}\left(\frac{273}{n}\right) \frac{\sum_{k=1}^{20}\left(\frac{-20}{k}\right) q^{k n}}{1-q^{20 n}}+\sum_{n=1}^{\infty}\left(\frac{-260}{n}\right) \frac{\sum_{k=1}^{21}\left(\frac{21}{k}\right) q^{k n}}{1-q^{21 n}}
\end{array}
$$




$$
\begin{aligned}
& +\sum_{n=1}^{\infty}\left(\frac{-195}{n}\right) \frac{\sum_{k=1}^{28}\left(\frac{28}{k}\right) q^{k n}}{1-q^{28 n}}-\sum_{n=1}^{\infty}\left(\frac{156}{n}\right) \frac{\sum_{k=1}^{35}\left(\frac{-35}{k}\right) q^{k n}}{1-q^{35 n}} \\
& -\sum_{n=1}^{\infty}\left(\frac{140}{n}\right) \frac{\sum_{k=1}^{39}\left(\frac{-39}{k}\right) q^{k n}}{1-q^{39 n}}-\sum_{n=1}^{\infty}\left(\frac{105}{n}\right) \frac{\sum_{k=1}^{52}\left(\frac{-52}{k}\right) q^{k n}}{1-q^{52 n}} \\
& \left.-\sum_{n=1}^{\infty}\left(\frac{-91}{n}\right) \frac{\sum_{k=1}^{60}\left(\frac{60}{k}\right) q^{k n}}{1-q^{60 n}}+\sum_{n=1}^{\infty}\left(\frac{-84}{n}\right) \frac{\sum_{k=1}^{65}\left(\frac{65}{k}\right) q^{k n}}{1-q^{65 n}}\right) .
\end{aligned}
$$

Acknowledgements. The author thanks Professor S. H. Chan for bringing to his attention the paper of Berkovich and Yesilyurt during a Number Theory seminar at the National University of Singapore. He also thanks Professor H. H. Chan for his guidance in this work. H. H. Chan's interpretation of $(1.1)$ and $(1.2)$ in the form $(1.3)$ and $(1.4)$ provided the motivation behind the discovery of the identities presented in this paper. Last but not least, the author thanks the anonymous referee for providing helpful suggestions.

\section{References}

[1] T. M. Apostol, Introduction to Analytic Number Theory, Undergrad. Texts Math., Springer, 1976.

[2] A. Berkovich and H. Yesilyurt, Ramanujan's identities and representation of integers by certain binary and quaternary quadratic forms, Ramanujan J. 20 (2009), 375-408.

[3] H. H. Chan and S. H. Chan, private communication.

[4] S. Chowla, An extension of Heilbronn's class number theorem, Quart. J. Math. (Oxford) 5 (1934), 304-307.

[5] S. Chowla and W. E. Briggs, On discriminants of binary quadratic forms with a single class in each genus, Canad. J. Math. 6 (1954), 463-470.

[6] D. A. Cox, Primes of the Form $x^{2}+n y^{2}$, Wiley, 1989.

[7] E. Grosswald, Negative discriminants of binary quadratic forms with one class in each genus, Acta Arith. 8 (1963), 295-306.

[8] N. A. Hall, The number of representations function for binary quadratic forms, Amer. J. Math. 62 (1940), 589-598.

[9] K. Ireland and M. Rosen, A Classical Introduction to Modern Number Theory, 2nd ed., Grad. Texts in Math. 84, Springer, 1990.

[10] P. Kaplan and K. S. Williams, On the number of representations of a positive integer by a binary quadratic form, Acta Arith. 114 (2004), 87-98.

[11] E. Landau, Elementary Number Theory, Chelsea, New York, 1958.

[12] L. C. Shen, On a class of q-series related to quadratic forms, Bull. Inst. Math, Acad. Sinica 26 (1998), 111-126.

[13] C. L. Siegel, Advanced Analytic Number Theory, 2nd ed., Tata Inst. Fund. Res. Stud. Math. 9, Tata Inst. Fund. Res., Bombay, 1980.

[14] Z. H. Sun and K. S. Williams, On the number of representations of $n$ by $a x^{2}+b x y+$ $c y^{2}$, Acta Arith. 122 (2006), 101-171. 
[15] J. D. Swift, Note on discriminants of binary quadratic forms with one class in each genus, Bull. Amer. Math. Soc. 54 (1948), 560-561.

[16] P. J. Weinberger, Exponents of the class groups of complex quadratic fields, Acta Arith. 22 (1973), 117-124.

[17] K. S. Williams, Some Lambert series expansion of products of theta functions, Ramanujan J. 3 (1999), 367-384.

Pee Choon Toh

Department of Mathematics

National University of Singapore

Blk S17, 10 Lower Kent Ridge Road

Singapore 119076

E-mail: mattpc@nus.edu.sg

Received on 4.8.2008

and in revised form on 12.12.2009 